\title{
La structure des prestations des partenaires tarifaires est prête
}

\author{
Christian Oeschger \\ Expert, $\mathrm{FMH}$, division Médecine et tarifs ambulatoires
}

\begin{abstract}
La structure des prestations, en négociation depuis le début de l'année entre la FMH et ses partenaires tarifaires curafutura, H+ et la CTM, a été validée les 24 et 25 octobre 2018 par l’Assemblée des délégués et la Chambre médicale. Elle est donc prête à être remise au Conseil fédéral. Dans les prochaines semaines, la FMH et ses sociétés de discipline vont négocier les règles d'application et de facturation.
\end{abstract}

Lors de la votation générale du 9 juin 2016, la majorité des membres de la FMH ayant exprimé leur opinion a refusé la proposition de tarif ambulatoire ats-tms V1.0 que la FMH avait développée conjointement avec ses partenaires $\mathrm{H}+$ et la CTM pendant quatre ans. Même après ce rejet, la nécessité de réviser le tarif était incontestée. C'est pourquoi la FMH a décidé dès 2016 de reprendre la révision, tout d'abord uniquement à l'interne, sans y associer les partenaires tarifaires.

La première phase a consisté à mettre en place le projet TARCO interne à la FMH dans le but d'obtenir un consensus entre les différentes sociétés de discipline médicale et associations faîtières et de disposer ensuite d'une base pour négocier avec les partenaires tarifaires. La structure tarifaire TARCO a été finalisée à la fin mars 2018 et approuvée à l'unanimité lors de l'Assemblée des délégués extraordinaire du 11 avril 2018 et

La structure des prestations, points tarifaires inclus, a franchi une étape supplémentaire.

de la Chambre médicale extraordinaire du 2 mai 2018. En parallèle, les négociations avec les partenaires tarifaires (phase 2) ont commencé fin 2017 en vue d'obtenir une proposition commune de tarif ambulatoire. Les résultats du projet TARCO ont fait partie des négociations avec les partenaires tarifaires et ont été en grande partie repris avec succès dans la structure des prestations négociée.

Les négociations entre les partenaires tarifaires impliqués (curafutura, H+, la CTM et la FMH) ont été finalisées fin septembre 2018 et une étape supplémentaire a été franchie lorsque l'Assemblée des délégués de la FMH et la Chambre médicale ont validé la structure des prestations révisée, points tarifaires inclus, les
24 et 25 octobre derniers. Les principales modifications par rapport au tarif actuellement en vigueur (TARMED 01.09.00_BR) vous sont présentées ci-après. La fin de l'article récapitule les prochaines étapes du projet de révision.

\section{Actualisation des modèles de coûts}

Les coûts d'utilisation des installations, et plus particulièrement des appareils, sont restés constants ou ont légèrement baissé (de nombreux appareils ont une fonctionnalité élargie mais leur prix n’a pas baissé de manière significative) alors que les frais de personnel ont notablement augmenté. D’après les estimations de la Caisse des médecins et de la FMH fondées sur l'étude permanente des coûts (RoKo), les coûts globaux des cabinets ont augmenté de près de $30 \%$ ces dernières années. Un objectif important de la révision a donc été de les recalculer selon les règles applicables en économie d'entreprise et de les intégrer ensuite dans les positions tarifaires.

Pour cela, les experts tarifaires ont alimenté les trois modèles de coûts usuels prestation médicale, KOREG et INFRA avec des données actualisées, et calculé les points tarifaires de la prestation médicale (PM) et de la prestation d'infrastructure et de personnel (PIP, anciennement prestation technique [PT]).

Pour l'actualisation de la PM, la FMH avait déposé la demande au conseil d'administration de l'ats-tms SA de reprendre le revenu de référence de CHF 229397 (actuellement CHF 207000) conformément à l'étude de l'Institut d'économie de la santé de Winterthour (WIG) financée par la FMH [1]. En début d'année, le conseil d'administration de l'ats-tms SA a décidé la reprise de ce revenu de référence. 
Pour le calcul des taux de coûts du KOREG, il a été possible d'utiliser les données actualisées de l'étude RoKo (2014-2016). Par rapport à la révision tarifaire de 2016, ces données sont devenues encore plus représentatives, 500 cabinets médicaux supplémentaires ayant pris part à cette étude. Les taux de coûts ont été calculés sur la base des données empiriques de l'étude et actualisés en conséquence. De plus, les données de

Les taux de coûts ont été calculés sur la base des données empiriques de l'étude RoKo et actualisés en conséquence.

l'étude RoKo concordent parfaitement avec les données de l'Office fédéral de la statistique (OFS) collectées dans le cadre du relevé «Données structurelles des cabinets médicaux et des centres de soins ambulatoires» (MAS).

Avec le modèle de coûts INFRA, il s'est agi de mettre l'accent sur les unités fonctionnelles au volume important, les fameuses Fokussparten (entre autres, radiologie, cardiologie). Elles ont été vérifiées en raison de leurs volumes de facturation et taux de coûts élevés puis réexaminées avec les partenaires tarifaires sous l'angle des dotations en appareils et en personnel. Pour cela, il a été demandé aux sociétés de discipline concernées de remettre factures et emplois du temps de collaborateurs concrets.

\section{Simplification et actualisation de la structure des prestations}

Malgré la création de nouvelles positions tarifaires dans de nombreux domaines, il a été possible de passer de 4500 à près de 2700 positions. Les positions existantes ont été examinées pour établir si elles étaient toujours à jour et répondaient encore aux critères médicaux actuels. Rappelons que la structure révisée comporte exclusivement les prestations fournies au cabinet.

\section{Interprétations générales}

Des modifications ont aussi été apportées dans les interprétations générales qui s'appliquent à l'ensemble de la structure des prestations. Les jeux de matériel dont le prix d'achat global dépasse CHF 3 pourront être facturés avec le tarif révisé pour autant que le prix d'achat d'au moins un élément dépasse $\mathrm{CHF} 3$ par pièce. Les partenaires tarifaires sont ouverts aux forfaits pour le matériel de certaines prestations dans des domaines comme la radiologie, la médecine nucléaire ou la radiothérapie.
La définition du fournisseur de prestations a également été modifiée. Les institutions au sens de l'art. 36a LAMal (institutions de soins ambulatoires dispensés par des médecins) sont considérées comme un seul et unique fournisseur de prestations, ce qui signifie que, pour chaque patient, les limitations s'appliquent à l'ensemble du cabinet de groupe. A l'hôpital, les limitations s'appliquent à l'ensemble des fournisseurs de prestations exécutant les traitements d'une même clinique spécialisée, institut ou centre au sein d'un hôpital.

Comme dans le TARMED 01.09.00_BR actuellement en vigueur, la documentation et la rédaction de rapports d'examen continuent de faire partie intégrante des positions tarifaires. La version en vigueur indemnise en partie une infrastructure surdotée. De nouvelles positions tarifaires ont été créées dans le chapitre AF.2O pour la rédaction de rapports à l'intention d'un autre médecin (p. ex. rapport de transfert), d'un patient ou d'un assureur. Ces rapports seront indemnisés selon le temps et non le nombre de lignes.

\section{Valeurs intrinsèques qualitatives}

Les valeurs intrinsèques qualitatives continuent de faire partie intégrante de la structure des prestations ambulatoires partenariale. Elles ont été remaniées en profondeur et actualisées en concertation avec les sociétés de discipline de la FMH.

\section{Valeurs intrinsèques quantitatives}

Après le nivellement des valeurs intrinsèques quantitatives suite à l'intervention du Conseil fédéral, ces dernières ont de facto disparu depuis le $1^{\text {er }}$ janvier 2018. Par conséquent, il est désormais devenu extrêmement difficile de les reprendre dans le tarif. Entretemps,

\section{La direction du GT valeurs intrinsèques ne} poursuit plus pour l'instant le projet de valeur intrinsèque médicale individuelle.

la direction du groupe de travail composé des quatre grandes organisations faîtières a décidé de ne pas poursuivre pour l'instant le projet de valeur intrinsèque médicale individuelle (VIMI). Les négociations sont encore en cours pour le supplément d'efficacité pour les prestations au temps.

Dans la structure tarifaire partenariale, les valeurs intrinsèques quantitatives sont de nouveau fixées à la valeur initiale de 1,0, à l'inverse du TARMED 01.09.00 BR, dans lequel elles sont fixées à 0,985. 


\section{Positions dites trigger et périodes d'une minute}

A l'inverse du TARMED 01.09.00_BR, les prestations au temps sont tarifées à la minute dans le nouveau tarif partenarial, à l'exception des quelques prestations du chapitre Prestations médicales de base (AF) qui disposent d'un socle initial de 5 minutes et sont ensuite facturées à la minute. Ces prestations constituent pour

\section{Avantage important: les 5 dernières minutes ne sont plus divisées par deux. Le temps effectif peut donc être entièrement facturé.}

les répondants des coûts ce qu'on appelle les positions trigger et font partie des caractéristiques d'une séance de consultation.

Le passage à des périodes de 1 minute n’a aucun impact sur le nombre de points tarifaires. Mais par rapport au système actuel, il apporte un avantage important: les 5 dernières minutes ne sont plus divisées par deux. Le temps effectif peut donc être entièrement facturé.

\section{Temps de changement, temps de pré- paration et de finition}

Le temps de changement et le temps de préparation et de finition sont repris dans la structure des prestations ambulatoires actualisée par le biais de positions tarifaires séparées. Ils sont ainsi toujours facturés dès qu'ils entrent en ligne de compte. Cela permet en même temps de ne pas les facturer plusieurs fois lorsque plusieurs prestations sont fournies dans la même infrastructure.

\section{Prestations médicales de base}

Les nombreux retours reçus des sociétés de discipline mais aussi de la part des répondants des coûts ont incité les partenaires à revoir les simplifications rigoureuses faites initialement dans ce chapitre. La critique principale résidait notamment dans la concentration radicale qui ne permettait plus de différencier suffisamment entre médecins et spécialisations en cas de possibles procédures EAE. La simplification a donc été en partie annulée au détriment de la transparence régulièrement exigée.

La proposition définitive continue de distinguer entre une consultation, une visite, une séance de télémédecine ou un consilium. Il existe désormais différents rapports médicaux et des prestations différentes en l'absence du patient. Les positions spécialisées créées dans de nombreux domaines ont une valeur identique (taux de coût identique à la minute) mais elles permettent de différencier les prestations médicales de base. Ces différenciations revêtent une importance primordiale pour les sociétés de discipline qui facturent une grande partie de leurs prestations via les positions de consultation.

\section{Processus opératoires et anesthésiques}

Dans le domaine des processus opératoires et anesthésiques, la nouvelle tarification a apporté de nombreuses modifications. La fmCh et la FMH y ont travaillé intensivement et elles ont discuté et développé plusieurs concepts différents. Fin 2017, les experts, sous la houlette de la fmCh, se sont mis d'accord sur les points suivants: Répartition fixe des opérations entre OP A (infrastructure installée, basse, correspondant à la salle d'opération au cabinet actuelle) ou OP B (infrastructure installée, moyenne, correspondant à la salle d'opération OP 1 actuelle).

Possibilités de mises à niveau pour garantir le décloisonnement entre les unités fonctionnelles, avant tout aussi pour la chirurgie pédiatrique: lorsque c'est médicalement indiqué et pertinent (et que l'unité fonctionnelle est reconnue), il sera donc possible d'exécuter une opération tarifée p. ex. en UBR Chirurgie dans une autre catégorie (OP B). Dans ce cas, le fournisseur de prestations doit aussi être indemnisé dans l'infrastructure OP B plus élevée. Des mises à niveau similaires sont prévues d'OP A à OP B et de OP B à OP C respectivement à OP D (bloc OP hybride).

Toutes les positions tarifaires opératoires se voient désormais attribuer ce qu'on appelle une classe d'intervention (Interventions-Aufwandsklasse [IAK]). Cela garantit un certain décloisonnement dans ce domaine. Ainsi les interventions sur les enfants de moins de 2ans sont automatiquement au moins en IAK III. Des condi-

\section{Toutes les positions tarifaires opératoires se} voient désormais attribuer ce qu'on appelle une classe d'intervention.

tions spéciales s'appliquent aussi pour les enfants jusqu'à 7 ans et les personnes âgées.

Création de prestations pour l'indemnisation des prestations générales de base en salle d'opération avant et après une opération visant à remplacer les prestations couvrant le sign in, le positionnement du patient, la préparation, le time out, le sign out, etc. Il existe cinq prestations générales de base différentes (IAK, MAC, I-IV) pour chaque unité fonctionnelle opératoire, qui se différencient par leur minutage. 


\section{Maintenance continue du tarif}

L'ats-tms SA est opérationnelle depuis début 2016 et les négociations ont eu lieu au sein de cette société. Le conseil d'administration réunit un nombre paritaire de représentants des fournisseurs de prestations et des

\section{Il existe cinq prestations générales de base différentes pour chaque unité fonctionnelle opératoire.}

assureurs. Les mises à jour se décident à la majorité, et non à l'unanimité comme dans l'ancienne organisation. Cela permet d'éviter les blocages par un seul et unique partenaire.

La société ats-tms SA va s'investir pour que la maintenance de la structure tarifaire soit garantie à l'avenir et créer pour cela un secrétariat opérationnel. De la sorte, la structure tarifaire ambulatoire révisée pose les jalons pour que le tarif soit adapté et actualisé chaque année. Ce suivi régulier se fonde sur un guide de tarification complet définissant et fixant les processus, les responsabilités et les organes.

Grâce à la structure des prestations négociée, les partenaires tarifaires impliqués peuvent retrouver l'autonomie tarifaire et la garantir. En revanche, les mesures supplémentaires de la Confédération, actuellement en discussion, ne sont plus nécessaires.

\section{Neutralité des coûts}

FMH / division Médecine

et tarifs ambulatoires

Baslerstrasse 47

CH-4600 Olten

Tél. 0313591230

Fax 0313591238

tarife.ambulant[at]fmh.ch

Conformément au résultat de la votation générale, la FMH ne poursuit pas une mise en œuvre neutre en termes de coûts de la révision mais s'en tient strictement aux dispositions de l'art. 43 al. 4 LAMal.

\section{Prochaines étapes}

Les prochaines étapes avec les partenaires tarifaires sont les suivantes: fin 2018, il est prévu de transmettre au Conseil fédéral la structure des prestations, points tarifaires et modèles de coûts inclus, approuvée par les partenaires tarifaires de l'ats-tms, pour un examen préalable (étape 1).

Une $2^{\mathrm{e}}$ étape prévoit d'élaborer et négocier, en collaboration avec les sociétés de discipline, les règles de facturation pour chaque chapitre. Cette manière de procéder a été décidée en août 2018 par le conseil d'administration de l'ats-tms SA parce qu'il est apparu que le temps ne suffirait pas pour établir les règles d'application et de facturation avant fin 2018 et que ces dernières ne pouvaient être définies qu'avec la participation des délégués tarifaires des sociétés de discipline.

\section{Une entrée en vigueur est donc toujours possible au $1^{\text {er }}$ janvier 2020.}

Il est prévu de remettre d'ici juin 2019 la structure tarifaire complète, finale et approuvée par les organes internes, incluant toutes les annexes et conventions ainsi que le système de règles au Conseil fédéral en tant que paquet global pour approbation définitive. Une entrée en vigueur est donc toujours possible au $1^{\text {er }}$ janvier 2020

\section{Référence}

1 Le revenu de référence réunit un salaire brut de CHF 196356 et les contributions sociales de l'employeur - que les médecins libéraux doivent verser eux-mêmes - à hauteur de CHF 33041. C'est une valeur mathématique qui ne reflète pas le revenu réel des médecins. Cf. l'article du BMS du 24 octobre 2018 sur le revenu des médecins. 